WITS-CTP-053

\title{
Gravitational Couplings of Higher Spins from String Theory
}

\author{
Dimitri Polyakov ${ }^{\dagger}$ \\ National Institute for Theoretical Physics (NITHeP) \\ and School of Physics \\ University of the Witwatersrand \\ WITS 2050 Johannesburg, South Africa
}

\begin{abstract}
We calculate the interaction 3-vertex of two massless spin 3 particles with a graviton using vertex operators for spin 3 fields in open string theory, constructed in our previous work. The massless spin 3 fields are shown to interact with the graviton through the linearized Weyl tensor, reproducing the result by Boulanger, Leclercq and Sundell. This is consistent with the general structure of the non-Abelian $2-s-s$ couplings, implying that the minimal number of space-time derivatives in the interaction vertices of two spin $\mathrm{s}$ and one spin 2 particle is equal to $2 s-2$.
\end{abstract}

May 2010

\footnotetext{
$\dagger$ dimitri.polyakov@wits.ac.za
} 


\section{Introduction}

Higher spin field theory is a fascinating subject that has attracted a lot of attention in recent years. Consistent description of theories with interacting higher spins is well-known to be a difficult problem, in particular because such theories need to involve powerful gauge symmetries, sufficient to remove the negative norm states. As a result, introducing higher spin interactions in a consistent way is a complicated issue with many challenging obstacles, despite certain progress in this direction over recent years [1], [2], [3], [4], [5], [6], [7], [8], [9], [10], [11], [12], [13], [14], [15], [16], [17], [18] String theory, on the other hand, appears to be a particularly efficient tool to describe interacting higher spins as many complex issues in higher spin field theories are settled very naturally in the string-theoretic approach. In particular, in our recent work [19] we have been able to construct the emission vertices for the massless higher spin fields with the integer spin values $3 \leq s \leq 9$. It has been shown that BRST-invariance conditions for these operators lead to standard Fierz-Pauli constraints on the space-time fields in the Fronsdal's approach [20]; BRSTnontriviality conditions, in turn, entail the gauge transformations for the space-time fields. The interaction terms of the higher spin fields could then be obtained from the scattering amplitudes on the string theory side; since the gauge transformations of the space-time fields shift the vertex operators by a trivial part (not contribuding to correlation functions), the interaction terms, obtained this way, will be gauge-invariant by construction.

In this work we concentrate on analyzing the important case of the spin 3 field's gravitational interaction, using the vertex operators constructed in our previous work. Since $s=3$ vertices are the open string operators, the spin 3 - graviton coupling is described by the appropriate disc amplitude in string theory, with two $s=3$ operators being on the boundary and the graviton in the bulk of the disc.

The amplitude, considered in this paper, is shown to reproduce some important properties of interacting higher spins, known from the field-theoretic approach [21], [22], [23], [24], 25]

In particular, while the gravitational couplings of lower spin fields contain at most two space-time derivatives in the Lagrangian, the gravitational couplings of the higher spin fields are known to have a very different behaviour [26], [27], [28], [22]. For example, there exists a unique nonabelian interaction 3-vertex of a graviton with 2 particles of spin $s$ containing $2 s-2$ space-time derivatives, related to the flat limit of the Fradkin-Vasiliev vertex in AdS. In a particularly important $s=3$ case the relation to the Fradkin-Vasiliev vertex can be established explicitly [21], [22] and the interaction of two $s=3$ particles with 
a graviton can be expressed in terms of linearized Weyl tensor [21], 22]. The purpose of this paper is to obtain this interaction from the string theory side, using the vertex operators constructed in our previous work. Computing the disc amplitude of two $s=3$ vertex operator with a graviton, we recover the structure of the gravitational coupling of massless spin 3 particles established in [21], 22], including the appearance of the Weyl tensor. The answer for the cubic interaction vertex that follows from the string theory computation, agrees with the result of [21], [22], modulo overall normalization coefficient and partial gauge fixing. The rest of the paper is organized as follows. In the Section 2 we recall the basic properties of the higher spin vertex operators, including the homotopy transformations needed to construct these operators at positive ghost pictures. In section 3 we perform the computation of the disc amplitude, showing the resulting interaction to agree with the coupling structure given in [21], [22]. In the concluding section we briefly discuss implications of our results, outlining future calculations.

\section{Computation of Spin 3 - Graviton Amplitude}

The vertex operator for a massless symmetric spin 3 particle is given by [19]:

$$
V_{s=3}(p)=H_{a_{1} a_{2} a_{3}}(p) c e^{-3 \phi} \partial X^{a_{1}} \partial X^{a_{2}} \psi^{a_{3}} e^{i \vec{p} \vec{X}}
$$

in the unintegrated $b-c$ picture and at the minimal negative $\beta-\gamma$ picture -3 . Here $H_{a_{1} a_{2} a_{3}}(p)$ is symmetric tensor satisfying the on-shell conditions

$$
\begin{array}{r}
p^{a_{1}} H_{a_{1} a_{2} a_{3}}(p)=0 \\
\eta^{a_{1} a_{2}} H_{a_{1} a_{2} a_{3}}(p)=0 \\
p^{2} H_{a_{1} a_{2} a_{3}}(p)=0
\end{array}
$$

as a consequence of the BRST-invariance constraints on the operator (1), while the gauge transformations

$$
H_{a_{1} a_{2} a_{3}}(p) \rightarrow H_{a_{1} a_{2} a_{3}}(p)+\partial_{\left(a_{1}\right.} \Lambda_{\left.a_{2} a_{3}\right)}
$$

with traceless symmetric $\Lambda$ parameter shift the vertex operator (1) by a trivial part, not contributing to correlators.

To compute a three-point amplitude of two spin 3 particles with a graviton one also needs the positive $\beta-\gamma$ picture +1 versions of $V_{s=3}$ in order to satisfy the ghost number anomaly cancellations. These operators exist at integrated $b-c$-picture only and can be obtained by replacing $-3 \phi \rightarrow \phi$ with the subsequent homotopy transformation described in [19], in order to ensure its BRST-invariance. As it has been shown [19], given the 
higher spin field described by ghost-dependent vertex operator at minimal negative superconformal ghost picture $-n-2(n \geq 1)$,

$$
V_{-n-2}=c e^{-(n+2) \phi} F_{\frac{n^{2}}{2}+n+1}(X, \psi)
$$

where (suppressing the space-time indices) $F_{\frac{n^{2}}{2}+n+1}(X, \psi)$ the is matter primary field of conformal dimension $\frac{n^{2}}{2}+n+1$. To construct the positive picture version of vertex operator describing such a state, one starts with constructing the charge

$$
\oint V_{n} \equiv \oint d z e^{n \phi} F_{\frac{n^{2}}{2}+n+1}(X, \psi)
$$

The BRST operator is given by

$$
Q_{b r s t}=Q_{1}+Q_{2}+Q_{3}
$$

where

$$
\begin{array}{r}
Q_{1}=\oint \frac{d z}{2 i \pi}\{c T-b c \partial c\} \\
Q_{2}=-\frac{1}{2} \oint \frac{d z}{2 i \pi} \gamma \psi_{a} \partial X^{a} \\
Q_{3}=-\frac{1}{4} \oint \frac{d z}{2 i \pi} b \gamma^{2}
\end{array}
$$

where $\mathrm{T}$ is the full stress-energy tensor. The operator (1) commutes with $Q_{1}$ since it is a worldsheet integral of dimension 1 and $b-c$ ghost number zero but doesn't commute with $Q_{2}$ and $Q_{3}$. To make it BRST-invariant, one has to add the correction terms by using the following procedure [29], [30]. We write

$$
\left[Q_{b r s t}, V_{n}(z)\right]=\partial U(z)+W_{1}(z)+W_{2}(z)
$$

and therefore

$$
\left[Q_{b r s t}, \oint d z V_{n}\right]=\oint d z\left(W_{1}(z)+W_{2}(z)\right)
$$

where

$$
\begin{aligned}
& U(z) \equiv c V_{n}(z) \\
& {\left[Q_{1}, V_{n}\right]=\partial U} \\
& W_{1}=\left[Q_{2}, V_{n}\right] \\
& W_{2}=\left[Q_{3}, V_{n}\right]
\end{aligned}
$$


Introduce the dimension $0 K$-operator:

$$
K(z)=-4 c e^{2 \chi-2 \phi}(z) \equiv \xi \Gamma^{-1}(z)
$$

satisfying

$$
\left\{Q_{b r s t}, K\right\}=1
$$

It is easy to check that this operator has a non-singular operator product with $W_{1}$ :

$$
K\left(z_{1}\right) W_{1}\left(z_{2}\right) \sim\left(z_{1}-z_{2}\right)^{2 n} Y\left(z_{2}\right)+O\left(\left(z_{1}-z_{2}\right)^{2 n+1}\right)
$$

where $Y$ is some operator of dimension $2 n+1$. Then the complete BRST-invariant operator can be obtained from $\oint d z V_{n}(z)$ by the following transformation:

$$
\begin{aligned}
\oint d z V_{n}(z) \rightarrow A_{n}(w)=\oint d z V_{n}(z) & +\frac{1}{(2 n) !} \oint d z(z-w)^{2 n}: K \partial^{2 n}\left(W_{1}+W_{2}\right):(z) \\
& +\frac{1}{(2 n) !} \oint d z \partial_{z}^{2 n+1}\left[(z-w)^{2 n} K(z)\right] K\left\{Q_{b r s t}, U\right\}
\end{aligned}
$$

where $w$ is some arbitrary point on the worldsheet. It is then straightforward to check the invariance of $A_{n}$ by using some partial integration along with the relation (12) as well as the obvious identity

$$
\left\{Q_{b r s t}, W_{1}(z)+W_{2}(z)\right\}=-\partial\left(\left\{Q_{b r s t}, U(z)\right\}\right)
$$

Although the invariant operators $A_{n}(w)$ depend on an arbitrary point $w$ on the worldsheet, this dependence is irrelevant in the correlators since all the $w$ derivatives of $A_{n}$ are BRST exact - the triviality of the derivatives ensures that there will be no $w$-dependence in any correlation functions involving $A_{n}$. Equivalently, the positive picture representations $A_{n}$ (14) for higher spin operators can also be obtained from minimal negative picture representations $V_{-n-2}$ by straightforward, but technically more cumbersome procedure by using the combination of the picture-changing and the $Z$-transformation (the analogue of the picture-changing for the $b-c$-ghosts).

Namely, the $Z$-operator, transforming the $b-c$ pictures (in particular, mapping integrated vertices to unintegrated) given by 31]

$$
Z(w)=b \delta(T)(w)=\oint d z(z-w)^{3}\left(b T+4 c \partial \xi \xi e^{-2 \phi} T^{2}\right)(z)
$$


where $T$ is the full stress-energy tensor in RNS theory. The usual picture-changing operator, transforming the $\beta-\gamma$ ghost pictures, is given by $\Gamma(w)=: \delta(\beta) G:(w)=: e^{\phi} G:(w)$. Introduce the integrated picture-changing operators $R_{n}(w)$ according to

$$
R_{n}(w)=Z(w): \Gamma^{n}:(w)
$$

where $: \Gamma^{n}$ : is the $n$th power of the standard picture-changing operator:

$$
\begin{aligned}
: \Gamma^{n}:(w) & =: e^{n \phi} \partial^{n-1} G \ldots \partial G G:(w) \\
& \equiv: \partial^{n-1} \delta(\beta) \ldots \partial \delta(\beta) \delta(\beta):
\end{aligned}
$$

Then the positive picture representations for the higher spin operators $A_{n}$ can be obtained from the negative ones $V_{-n-2}(1)$ by the transformation:

$$
A_{n}(w)=\left(R_{2}\right)^{n+1}(w) V_{-n-2}(w)
$$

Since both $Z$ and $\Gamma$ are BRST-invariant and nontrivial, the $A_{n}$-operators by construction satisfy the BRST-invariance and non-triviality conditions identical to those satisfied by their negative picture counterparts $V_{-2 n-2}$ and therefore lead to the same Pauli-Fierz on-shell conditions and the gauge symmetries for the higher spin fields. Applying the above procedure to the $s=3$ vertex operator (1) (corresponding to the $n=2$ case) we obtain the following expression for the positive +1 picture operators for $s=3$ massless particles.

$$
A_{s=3}(w)=\oint d z(z-w)^{2} U(z) \equiv A_{0}+A_{1}+A_{2}+A_{3}+A_{4}+A_{5}+A_{6}+A_{7}+A_{8}
$$

where

$$
A_{0}(w)=\frac{1}{2} H_{a_{1} a_{2} a_{3}}(p) \oint d z(z-w)^{2} P_{2 \phi-2 \chi-\sigma}^{(2)} e^{\phi} \partial X^{a_{1}} \partial X^{a_{2}} \psi^{a_{3}} e^{i \vec{p} \vec{X}}(z)
$$

and

$$
\left.A_{8}(w)=-12 H_{a_{1} a_{2} a_{3}}(p) \oint d z(z-w)^{2} \partial c c \partial \xi \xi e^{-\phi} \partial X^{a_{1}} \partial X^{a_{2}} \psi^{a_{3}}\right\} e^{i \vec{p} \vec{X}}(z)
$$


have ghost factors proportional to $e^{\phi}$ and $\partial c c \partial \xi \xi e^{-\phi}$ respectively and the rest of the terms carry ghost factor proportional to $c \xi$ :

$$
\begin{array}{r}
A_{1}(w)=-2 H_{a_{1} a_{2} a_{3}}(p) \oint d z(z-w)^{2} c \xi(\vec{\psi} \partial \vec{X}) \partial X^{a_{1}} \partial X^{a_{2}} \psi^{a_{3}} e^{i \vec{p} \vec{X}}(z) \\
A_{2}(w)=-H_{a_{1} a_{2} a_{3}}(p) \oint d z(z-w)^{2} c \xi \partial X^{a_{1}} \partial X^{a_{2}} \partial X^{a_{3}} P_{\phi-\chi}^{(1)} e^{i \vec{p} \vec{X}}(z) \\
A_{3}(w)=-H_{a_{1} a_{2} a_{3}}(p) \oint d z(z-w)^{2} c \xi \partial X^{a_{1}} \partial X^{a_{2}} \partial^{2} X^{a_{3}} e^{i \vec{p} \vec{X}}(z) \\
\left.A_{4}(w)=2 H_{a_{1} a_{2} a_{3}}(p) \oint d z(z-w)^{2} c \xi \partial \psi^{a_{2}} P_{\phi-\chi}^{(1)}\right) \psi^{a_{3}} e^{i \vec{p} \vec{X}}(z) \\
A_{5}(w)=2 H_{a_{1} a_{2} a_{3}}(p) \oint d z(z-w)^{2} c \xi \partial^{2} \psi^{a_{2}} \psi^{a_{3}} e^{i \vec{p} \vec{X}}(z) \\
A_{5}(w)=-2 H_{a_{1} a_{2} a_{3}}(p) \oint d z(z-w)^{2} c \xi \partial X^{a_{1}} \partial X^{a_{2}}\left(\partial^{2} X^{a_{3}}+\partial X^{a_{3}} P_{\phi-\chi}^{(1)}\right) e^{i \vec{p} \vec{X}}(z) \\
A_{6}(w)=2 i H_{a_{1} a_{2} a_{3}}(p) \oint d z(z-w)^{2} c \xi(\vec{p} \vec{\psi}) P_{\phi-\chi}^{(1)} \partial X^{a_{1}} \partial X^{a_{2}} \psi^{a_{3}} e^{i \vec{p} \vec{X}}(z) \\
A_{7}(w)=2 i H_{a_{1} a_{2} a_{3}}(p) \oint d z(z-w)^{2} c \xi(\vec{p} \partial \vec{\psi}) \partial X^{a_{1}} \partial X^{a_{2}} \psi^{a_{3}} e^{i \vec{p} \vec{X}}(z)
\end{array}
$$

\section{Calculation of the Disc Amplitude}

To evaluate the three-point amplitude of two $s=3$ particles with a graviton on the disc, it is convenient to take both of the $s=3$ vertices at positive +1 -picture (integrated over the disc boundary), while taking the graviton at the disc origin unintegrated and at the left and right $\beta-\gamma$ ghost pictures -1 and -2 respectively:

$$
V_{s=2}=\gamma_{m_{1} m_{2}}(p) c \bar{c} e^{-\phi-2 \bar{\phi}} \psi^{m_{1}} \bar{\partial} X^{m_{2}}(0,0)
$$

Furthermore, it shall be convenient to make conformal mapping from the disc to the upper half-plane $(z, \bar{z}) \rightarrow(w, \bar{w})$ using $w=-i \frac{z-i}{z+i}$ so the the graviton's location is mapped to $w=i$. As the ghost number anomaly cancellation requires that the the total $\phi$-ghost number of correlation functions has to be equal to $-2, \chi$-ghost number equal to 1 and the $\sigma$-ghost number equal to 3 , the three-point correlator $\left\langle A_{s=3}\left(w_{1}\right) A_{s=3}\left(w_{2}\right) A_{s=2}(i)\right\rangle$ is contributed by the terms

$$
<A_{s=3}\left(w_{1}\right) A_{s=3}\left(w_{2}\right) A_{s=2}(i)>=\sum_{j=1}^{7}<A_{j}\left(w_{1}\right) A_{0}\left(w_{2}\right) A_{s=2}(i)>
$$

of (21), (23), (24). So we shall calculate these terms one by one, using (23). The cubic interaction vertex is determined by the structure constants given by the on-shell limit of 
(25), implying $\left(p_{i} p_{j}\right)=0$ In this paper, we are interested in the contributions to the correlator (25) up to the terms quartic in momentum. More precisely, our main result is that all the terms that are zero or quadratic in momentum vanish (as it is immediately clear from the structure of the operators $(21),(23),(24)$ that there are no terms linear or cubic in $p$ ) and the lowest order terms are thus quartic in momentum (and hence in space-time derivatives). As for the quartic order terms, we shall show that they combine into the cubic interaction vertex manifestly dependent on the linearized Weyl tensor, in agreement with the results of [21], [22].

To start with, it is convenient to point out the ghost factor, common for all the terms in (25). It is given by:

$$
\begin{array}{r}
G_{g h}\left(z_{1}, z_{2}, z, \bar{z}\right)=<c e^{\chi}\left(w_{1}\right) P_{2 \phi-2 \chi-\sigma}^{(2)} e^{\phi}\left(w_{2}\right) c e^{-\phi}(z) \bar{c} e^{-2 \bar{\phi}}(\bar{z})> \\
=\frac{\left(z-z_{1}\right)\left(z-z_{2}\right)\left(\bar{z}-z_{1}\right)\left(\bar{z}-z_{2}\right)^{2}}{(z-\bar{z})} \\
\times\left\{\frac{2}{\left(z_{1}-z_{2}\right)^{2}}+\frac{1}{\left(\bar{z}-z_{2}\right)^{2}}+\frac{1}{\left(z-z_{2}\right)\left(\bar{z}-z_{2}\right)}-\frac{1}{\left(z-z_{2}\right)\left(z_{1}-z_{2}\right)}-\frac{3}{\left(z_{1}-z_{2}\right)\left(\bar{z}-z_{2}\right)}\right\}
\end{array}
$$

Since the correlation functions of the spin 3 operators $A(w) \sim \int d z(z-w)^{2} U(z)$ do not depend on $w$,it is convenient to choose $w_{1,2}= \pm i$ for the first and for the second $s=$ 3 operators. We start from the contributions to (25), quartic in the momentum. The calculation of the first contribution, using the expressions (23), (24) gives

$$
\begin{array}{r}
<A_{1}(i) A_{0}(-i) V_{s=2}(z,-\bar{z})>_{4}=H_{a_{1} a_{2} a_{3}}\left(p_{1}\right) H_{b_{1} b_{2} b_{3}}\left(p_{2}\right) \gamma_{m_{1} m_{2}}\left(p_{3}\right) \\
\times \int_{-\infty}^{\infty} d z_{1} \int_{-\infty}^{\infty} d z_{2}\left(z_{1}-i\right)^{2}\left(z_{2}+i\right)^{2} G_{g h}\left(z_{1}, z_{2}, i,-i\right) \\
\times\left\{-\frac{\eta^{a_{4} b_{2}}\left(-\eta^{a_{4} b_{3}} \eta^{a_{3} m_{2}}+\eta^{a_{3} b_{3}} \eta^{a_{4} m_{2}}\right)}{\left(z_{1}-z_{2}\right)^{2}\left(z_{1}-z\right)\left(z_{2}-\bar{z}\right)} p_{2}^{a_{1}} p_{2}^{a_{2}} p_{1}^{b_{1}} p_{1}^{m_{1}}\right. \\
\times\left(\frac{1}{z_{1}-z_{2}}-\frac{1}{z_{1}-\bar{z}}\right)^{2}\left(\frac{1}{z_{1}-z_{2}}+\frac{1}{z_{2}-\bar{z}}\right)\left(\frac{1}{z_{1}-\bar{z}}-\frac{1}{z_{2}-\bar{z}}\right) \\
-\frac{\eta^{a_{4} m_{1}}\left(-\eta^{a_{4} b_{3}} \eta^{a_{3} m_{2}}+\eta^{a_{3} b_{3}} \eta^{a_{4} m_{2}}\right)}{\left(z_{1}-\bar{z}\right)^{2}\left(z_{1}-z\right)\left(z_{2}-\bar{z}\right)} p_{2}^{a_{1}} p_{2}^{a_{2}} p_{1}^{b_{1}} p_{1}^{b_{2}}\left(\frac{1}{z_{1}-z_{2}}-\frac{1}{z_{1}-\bar{z}}\right)^{2}\left(\frac{1}{z_{1}-z_{2}}+\frac{1}{z_{2}-\bar{z}}\right)^{2} \\
\left.-\frac{\eta^{b_{2} m_{1}}\left(-\eta^{a_{4} b_{3}} \eta^{a_{3} m_{2}}+\eta^{a_{3} b_{3}} \eta^{a_{4} m_{2}}\right)}{\left(z_{2}-\bar{z}\right)^{2}\left(z_{1}-\bar{z}\right)\left(z_{2}-z\right)} p_{2}^{a_{1}} p_{2}^{a_{2}} p_{2}^{a_{4}} p_{1}^{b_{1}}\left(\frac{1}{z_{1}-z_{2}}-\frac{1}{z_{1}-\bar{z}}\right)^{3}\left(\frac{1}{z_{1}-z_{2}}+\frac{1}{z_{2}-\bar{z}}\right)\right\}
\end{array}
$$

Here and everywhere below $z, \bar{z} \equiv \pm i$. The next contribution to the amplitude part, quartic 
in momentum, is given by

$$
\begin{array}{r}
<A_{2}(i) A_{0}(-i) V_{s=2}(z, \bar{z})>_{4}=H_{a_{1} a_{2} a_{3}}\left(p_{1}\right) H_{b_{1} b_{2} b_{3}}\left(p_{2}\right) \gamma_{m_{1} m_{2}}\left(p_{3}\right) \\
\times \int_{-\infty}^{\infty} d z_{1} \int_{-\infty}^{\infty} d z_{2}\left(z_{1}-i\right)^{2}\left(z_{2}+i\right)^{2} G_{g h}\left(z_{1}, z_{2}, i,-i\right)\left(-\frac{1}{z_{1}-z_{2}}+\frac{2}{z_{1}-\bar{z}}+\frac{1}{z_{1}-z}\right) \\
\times\left\{\frac{\eta^{b_{3} m_{2}} \eta^{a_{3} b_{2}}}{\left(z_{1}-z_{2}\right)^{2}\left(z-z_{2}\right)} p_{2}^{a_{1}} p_{2}^{a_{2}} p_{1}^{b_{1}} p_{1}^{m_{1}}\left(\frac{1}{z_{1}-z_{2}}-\frac{1}{z_{1}-\bar{z}}\right)^{2}\right. \\
\times\left(\frac{1}{z_{1}-z_{2}}+\frac{1}{z_{2}-\bar{z}}\right)\left(-\frac{1}{z_{1}-\bar{z}}-\frac{1}{z_{2}-\bar{z}}\right) \\
+\frac{\eta^{b_{3} m_{2}} \eta^{a_{3} m_{1}}}{\left(z_{1}-\bar{z}\right)^{2}\left(z-z_{2}\right)} p_{2}^{a_{1}} p_{2}^{a_{2}} p_{1}^{b_{1}} p_{1}^{b_{2}}\left(\frac{1}{z_{1}-z_{2}}-\frac{1}{z_{1}-\bar{z}}\right)^{2}\left(\frac{1}{z_{1}-z_{2}}+\frac{1}{z_{2}-\bar{z}}\right)^{2} \\
\left.-\frac{\eta^{b_{3} m_{2}} \eta^{b_{2} m_{1}}}{\left(z_{2}-\bar{z}\right)^{2}\left(z-z_{2}\right)} p_{2}^{a_{1}} p_{2}^{a_{2}} p_{2}^{a_{3}} p_{1}^{b_{1}}\left(\frac{1}{z_{1}-z_{2}}-\frac{1}{z_{1}-\bar{z}}\right)^{3}\left(\frac{1}{z_{1}-z_{2}}+\frac{1}{z_{2}-\bar{z}}\right)\right\}
\end{array}
$$

The next contribution is

$$
\begin{aligned}
& <A_{3}(i) A_{0}(-i) V_{s=2}(z, \bar{z})>_{4}=H_{a_{1} a_{2} a_{3}}\left(p_{1}\right) H_{b_{1} b_{2} b_{3}}\left(p_{2}\right) \gamma_{m_{1} m_{2}}\left(p_{3}\right) \\
& \times \int_{-\infty}^{\infty} d z_{1} \int_{-\infty}^{\infty} d z_{2}\left(z_{1}-i\right)^{2}\left(z_{2}+i\right)^{2} G_{g h}\left(z_{1}, z_{2}, i,-i\right) \\
& \times\left\{\frac { \eta ^ { b _ { 3 } m _ { 2 } } \eta ^ { a _ { 3 } b _ { 2 } } } { ( z - z _ { 2 } ) } p _ { 2 } ^ { a _ { 1 } } p _ { 2 } ^ { a _ { 2 } } p _ { 1 } ^ { b _ { 1 } } p _ { 1 } ^ { m _ { 1 } } \partial _ { z _ { 1 } } \left\{\frac{1}{\left(z_{1}-z_{2}\right)^{2}}\left(\frac{1}{z_{1}-z_{2}}-\frac{1}{z_{1}-\bar{z}}\right)^{2}\right.\right. \\
& \left.\times\left(\frac{1}{z_{1}-z_{2}}+\frac{1}{z_{2}-\bar{z}}\right)\left(-\frac{1}{z_{1}-\bar{z}}-\frac{1}{z_{2}-\bar{z}}\right)\right\} \\
& +\frac{\eta^{b_{3} m_{2}} \eta^{a_{3} m_{1}}}{\left(z-z_{2}\right)} p_{2}^{a_{1}} p_{2}^{a_{2}} p_{1}^{b_{1}} p_{1}^{b_{2}} \partial_{z_{1}}\left\{\frac{1}{\left(z_{1}-\bar{z}\right)^{2}}\left(\frac{1}{z_{1}-z_{2}}-\frac{1}{z_{1}-\bar{z}}\right)^{2}\left(\frac{1}{z_{1}-z_{2}}+\frac{1}{z_{2}-\bar{z}}\right)^{2}\right\} \\
& -\frac{\eta^{b_{3} m_{2}} \eta^{b_{2} m_{1}}}{\left(z-z_{2}\right)} p_{2}^{a_{1}} p_{2}^{a_{2}} p_{2}^{a_{3}} p_{1}^{b_{1}} \partial_{z_{1}}\left\{\frac{1}{\left(z_{2}-\bar{z}\right)^{2}}\left(\frac{1}{z_{1}-z_{2}}-\frac{1}{z_{1}-\bar{z}}\right)^{3}\left(\frac{1}{z_{1}-z_{2}}+\frac{1}{z_{2}-\bar{z}}\right)\right\}
\end{aligned}
$$

Next,

$$
\begin{array}{r}
<A_{4}(i) A_{0}(-i) V_{s=2}(z, \bar{z})>_{4}=2 H_{a_{1} a_{2} a_{3}}\left(p_{1}\right) H_{b_{1} b_{2} b_{3}}\left(p_{2}\right) \gamma_{m_{1} m_{2}}\left(p_{3}\right) \\
\times \int_{-\infty}^{\infty} d z_{1} \int_{-\infty}^{\infty} d z_{2}\left(z_{1}-i\right)^{2}\left(z_{2}+i\right)^{2} G_{g h}\left(z_{1}, z_{2}, i,-i\right)\left(-\frac{1}{z_{1}-z_{2}}+\frac{2}{z_{1}-\bar{z}}+\frac{1}{z_{1}-z}\right) \\
\times\left\{\left(-\frac{\eta^{a_{3} b_{3}} \eta^{a_{2} m_{2}}}{\left(z_{1}-z_{2}\right)\left(z_{1}-z\right)^{2}}+\frac{\eta^{a_{3} m_{2}} \eta^{a_{2} b_{3}}}{\left(z_{1}-z\right)\left(z_{1}-z_{2}\right)^{2}}\right) p_{2}^{a_{1}} p_{1}^{b_{1}} p_{1}^{b_{2}} p_{1}^{m_{1}}\right. \\
\left.\times\left(\frac{1}{z_{1}-z_{2}}-\frac{1}{z_{1}-\bar{z}}-\frac{1}{z_{1}-z}\right)\left(\frac{1}{z_{1}-z_{2}}+\frac{1}{z_{2}-\bar{z}}+\frac{1}{z_{2}-z}\right)^{2}\left(\frac{1}{z_{1}-\bar{z}}+\frac{1}{z_{2}-\bar{z}}\right)\right\}
\end{array}
$$

Next, 


$$
\begin{array}{r}
<A_{5}(i) A_{0}(-i) V_{s=2}(z, \bar{z})>_{4}=-H_{a_{1} a_{2} a_{3}}\left(p_{1}\right) H_{b_{1} b_{2} b_{3}}\left(p_{2}\right) \gamma_{m_{1} m_{2}}\left(p_{3}\right) \\
\times \int_{-\infty}^{\infty} d z_{1} \int_{-\infty}^{\infty} d z_{2}\left(z_{1}-i\right)^{2}\left(z_{2}+i\right)^{2} G_{g h}\left(z_{1}, z_{2}, i,-i\right) \\
\quad \times\left\{\left(-\frac{\eta^{a_{3} b_{3}} \eta^{a_{2} m_{2}}}{\left(z_{1}-z_{2}\right)\left(z_{1}-z\right)^{2}}\left(\frac{1}{z_{1}-z_{2}}+\frac{2}{z_{1}-z}\right)\right.\right. \\
\left.-\frac{\eta^{a_{3} m_{2}} \eta^{a_{2} b_{3}}}{\left(z_{1}-z\right)\left(z_{1}-z_{2}\right)^{2}}\left(\frac{1}{z_{1}-\bar{z}}+\frac{2}{z_{1}-z_{2}}\right)\right) p_{2}^{a_{1}} p_{1}^{b_{1}} p_{1}^{b_{2}} p_{1}^{m_{1}} \\
\left.\times\left(\frac{1}{z_{1}-z_{2}}-\frac{1}{z_{1}-\bar{z}}-\frac{1}{z_{1}-z}\right)\left(\frac{1}{z_{1}-z_{2}}+\frac{1}{z_{2}-\bar{z}}+\frac{1}{z_{2}-z}\right)^{2}\left(\frac{1}{z_{1}-\bar{z}}+\frac{1}{z_{2}-\bar{z}}\right)\right\}
\end{array}
$$

Next,

$$
\begin{aligned}
& <A_{6}(i) A_{0}(-i) V_{s=2}(z, \bar{z})>_{4}=H_{a_{1} a_{2} a_{3}}\left(p_{1}\right) H_{b_{1} b_{2} b_{3}}\left(p_{2}\right) \gamma_{m_{1} m_{2}}\left(p_{3}\right) \\
& \times \int_{-\infty}^{\infty} d z_{1} \int_{-\infty}^{\infty} d z_{2}\left(z_{1}-i\right)^{2}\left(z_{2}+i\right)^{2} G_{g h}\left(z_{1}, z_{2}, i,-i\right)\left(-\frac{1}{z_{1}-z_{2}}+\frac{2}{z_{1}-\bar{z}}+\frac{1}{z_{1}-z}\right) \\
& \times\left\{\frac { ( \eta ^ { a _ { 3 } b _ { 3 } } \eta ^ { a _ { 4 } m _ { 2 } } - \eta ^ { a _ { 3 } m _ { 2 } } \eta ^ { a _ { 2 } b _ { 3 } } ) ( p _ { 1 } ) _ { a _ { 4 } } } { ( z _ { 1 } - z _ { 2 } ) ( z _ { 1 } - z ) } \left[\frac{\eta^{a_{2} b_{2}}}{\left(z_{1}-z_{2}\right)^{2}} p_{2}^{a_{1}} p_{1}^{b_{1}} p_{1}^{m_{1}}\right.\right. \\
& \times\left(\frac{1}{z_{1}-z_{2}}-\frac{1}{z_{1}-\bar{z}}-\frac{1}{z_{1}-z}\right)\left(\frac{1}{z_{1}-z_{2}}+\frac{1}{z_{2}-\bar{z}}+\frac{1}{z_{2}-z}\right)\left(\frac{1}{z_{1}-\bar{z}}+\frac{1}{z_{2}-\bar{z}}\right) \\
& +\frac{\eta^{a_{2} m_{1}}}{\left(z_{1}-\bar{z}\right)^{2}} p_{2}^{a_{1}} p_{1}^{b_{1}} p_{1}^{b_{2}} \\
& \times\left(\frac{1}{z_{1}-z_{2}}-\frac{1}{z_{1}-\bar{z}}-\frac{1}{z_{1}-z}\right)\left(\frac{1}{z_{1}-z_{2}}+\frac{1}{z_{2}-\bar{z}}+\frac{1}{z_{2}-z}\right)^{2}-\frac{\eta^{b_{2} m_{1}}}{\left(z_{2}-\bar{z}\right)^{2}} p_{2}^{a_{1}} p_{2}^{a_{2}} p_{1}^{b_{1}} \\
& \left.\times\left(\frac{1}{z_{1}-z_{2}}-\frac{1}{z_{1}-\bar{z}}-\frac{1}{z_{1}-z}\right)^{2}\left(\frac{1}{z_{1}-z_{2}}+\frac{1}{z_{2}-\bar{z}}+\frac{1}{z_{2}-z}\right)\right\}
\end{aligned}
$$


Finally,

$$
\begin{array}{r}
<A_{7}(i) A_{0}(-i) V_{s=2}(z, \bar{z})>_{4}=H_{a_{1} a_{2} a_{3}}\left(p_{1}\right) H_{b_{1} b_{2} b_{3}}\left(p_{2}\right) \gamma_{m_{1} m_{2}}\left(p_{3}\right) \\
\times \int_{-\infty}^{\infty} d z_{1} \int_{-\infty}^{\infty} d z_{2}\left(z_{1}-i\right)^{2}\left(z_{2}+i\right)^{2} G_{g h}\left(z_{1}, z_{2}, i,-i\right)\left(-\frac{1}{z_{1}-z_{2}}+\frac{2}{z_{1}-\bar{z}}+\frac{1}{z_{1}-z}\right) \\
\times\left\{\left(\frac{\eta^{a_{3} b_{3}} \eta^{a_{4} m_{2}}}{\left(z_{1}-z_{2}\right)\left(z_{1}-z\right)^{2}}-\frac{\eta^{a_{3} m_{2}} \eta^{a_{2} b_{3}}}{\left(z_{1}-z_{2}\right)^{2}\left(z_{1}-z\right)}\right)\left(p_{1}\right)_{a_{4}}\right. \\
{\left[\frac{\eta^{a_{2} b_{2}}}{\left(z_{1}-z_{2}\right)^{2}} p_{2}^{a_{1}} p_{1}^{b_{1}} p_{1}^{m_{1}}\right.} \\
\times\left(\frac{1}{z_{1}-z_{2}}-\frac{1}{z_{1}-\bar{z}}-\frac{1}{z_{1}-z}\right)\left(\frac{1}{z_{1}-z_{2}}+\frac{1}{z_{2}-\bar{z}}+\frac{1}{z_{2}-z}\right)\left(\frac{1}{z_{1}-\bar{z}}+\frac{1}{z_{2}-\bar{z}}\right) \\
\times\left(\frac{\eta^{a_{2} m_{1}}}{\left(z_{1}-\bar{z}\right)^{2}} p_{2}^{a_{1}} p_{1}^{b_{1}} p_{1}^{b_{2}}\right. \\
\left.z_{1}-z_{2}-\frac{1}{z_{1}-\bar{z}}-\frac{1}{z_{1}-z}\right)\left(\frac{1}{z_{1}-z_{2}}+\frac{1}{z_{2}-\bar{z}}+\frac{1}{z_{2}-z}\right)^{2}-\frac{\eta^{b_{2} m_{1}}}{\left(z_{2}-\bar{z}\right)^{2}} p_{2}^{a_{1}} p_{2}^{a_{2}} p_{1}^{b_{1}} \\
\left.\times\left(\frac{1}{z_{1}-z_{2}}-\frac{1}{z_{1}-\bar{z}}-\frac{1}{z_{1}-z}\right)^{2}\left(\frac{1}{z_{1}-z_{2}}+\frac{1}{z_{2}-\bar{z}}+\frac{1}{z_{2}-z}\right)\right\}
\end{array}
$$

This concludes the list of the contributions, quartic in momentum, to the amplitude (25).

Next, we shall concentrate on the terms, quadratic in $p$. The straightforward evaluation gives

$$
\begin{array}{r}
<A_{1}(i) A_{0}(-i) V_{s=2}(z, \bar{z})>_{2}=-H_{a_{1} a_{2} a_{3}}\left(p_{1}\right) H_{b_{1} b_{2} b_{3}}\left(p_{1}\right) \gamma_{m_{1} m_{2}}\left(p_{2}\right) \\
\times \int_{-\infty}^{\infty} d z_{1} \int_{-\infty}^{\infty} d z_{2}\left(z_{1}-i\right)^{2}\left(z_{2}+i\right)^{2} G_{g h}\left(z_{1}, z_{2}, i,-i\right) \\
\times \frac{\eta^{a_{3} b_{3}} \eta^{a_{4} m_{2}}-\eta^{a_{4} b_{3}} \eta^{a_{4} m_{2}}}{\left(z_{1}-z\right)\left(z_{2}-\bar{z}\right)}\left\{p_{2}^{a_{1}} p_{2}^{a_{2}}\left(\frac{1}{z_{1}-z_{2}}-\frac{1}{z_{1}-\bar{z}}\right)^{2} \frac{\eta^{a_{4} b_{1}} \eta^{b_{2} m_{1}}}{\left(z_{1}-z_{2}\right)^{2}\left(z_{2}-\bar{z}\right)^{2}}\right. \\
-p_{2}^{a_{1}} p_{1}^{b_{1}}\left(\frac{1}{z_{1}-z_{2}}-\frac{1}{z_{1}-\bar{z}}-\frac{1}{z_{1}-z}\right)\left(\frac{1}{z_{1}-z_{2}}+\frac{1}{z_{2}-\bar{z}}+\frac{1}{z_{2}-z}\right) \frac{\eta^{a_{2} b_{2}} \eta^{a_{4} m_{1}}}{\left(z_{1}-z_{2}\right)^{2}\left(z_{2}-\bar{z}\right)^{2}} \\
\left.+p_{2}^{a_{1}} p_{1}^{b_{1}}\left(\frac{1}{z_{1}-z_{2}}-\frac{1}{z_{1}-\bar{z}}-\frac{1}{z_{1}-z}\right)\left(-\frac{1}{z_{1}-\bar{z}}+\frac{1}{z_{2}-\bar{z}}\right) \frac{\eta^{a_{2} b_{2}} \eta^{a_{4} m_{1}}}{\left(z_{1}-z_{2}\right)^{4}}\right\}
\end{array}
$$

The next contribution, quadratic in momentum, is given by 


$$
\begin{array}{r}
<A_{2}(i) A_{0}(-i) V_{s=2}(z, \bar{z})>_{2}=H_{a_{1} a_{2} a_{3}}\left(p_{1}\right) H_{b_{1} b_{2} b_{3}}\left(p_{2}\right) \gamma_{m_{1} m_{2}}\left(p_{3}\right) \\
\times \int_{-\infty}^{\infty} d z_{1} \int_{-\infty}^{\infty} d z_{2}\left(z_{1}-i\right)^{2}\left(z_{2}+i\right)^{2} G_{g h}\left(z_{1}, z_{2}, i,-i\right) \\
\times \frac{\eta^{b_{3} m_{2}}}{\left(z_{2}-z\right)}\left(-\frac{1}{z_{1}-z_{2}}+\frac{2}{z_{1}-\bar{z}}+\frac{1}{z_{1}-z}\right) \\
\times\left\{p_{2}^{a_{1}} p_{2}^{a_{2}} \frac{\eta^{a_{3} b_{1}} \eta^{b_{2} m_{1}}}{\left(z_{1}-z_{2}\right)^{2}\left(z_{2}-\bar{z}\right)^{2}}\left(\frac{1}{z_{1}-z_{2}}-\frac{1}{z_{1}-\bar{z}}-\frac{1}{z_{1}-z}\right)^{2}\right. \\
\times\left(\frac{1}{z_{1}-z_{2}}-\frac{1}{z_{1}-\bar{z}}-\frac{1}{z_{1}-z}\right)\left(\frac{1}{z_{1}-z_{2}}+\frac{1}{z_{2}-\bar{z}}+\frac{1}{z_{2}-z}\right) \\
-p_{2}^{a_{1}} p_{1}^{m_{1}} \frac{\eta^{a_{2} b_{2}} \eta^{a_{3} m_{1}} \eta^{a_{3} b_{2}}}{\left(z_{1}-z_{2}\right)^{4}}\left(\frac{1}{z_{1}-z_{2}}-\frac{1}{z_{1}-\bar{z}}-\frac{1}{z_{1}-z}\right) \\
\left.\times\left(\frac{1}{z_{1}-z_{2}}+\frac{1}{z_{2}-\bar{z}}+\frac{1}{z_{2}-z}\right)\left(-\frac{1}{z_{1}-\bar{z}}+\frac{1}{z_{2}-\bar{z}}\right)\right\}
\end{array}
$$

Next,

$$
\begin{array}{r}
<A_{3}(i) A_{0}(-i) V_{s=2}(z, \bar{z})>_{2}=H_{a_{1} a_{2} a_{3}}\left(p_{1}\right) H_{b_{1} b_{2} b_{3}}\left(p_{2}\right) \gamma_{m_{1} m_{2}}\left(p_{3}\right) \\
\times \int_{-\infty}^{\infty} d z_{1} \int_{-\infty}^{\infty} d z_{2}\left(z_{1}-i\right)^{2}\left(z_{2}+i\right)^{2} G_{g h}\left(z_{1}, z_{2}, i,-i\right) \\
\times \frac{\eta^{b_{3} m_{2}}}{\left(z_{2}-z\right)} \\
\times\left\{p_{2}^{a_{1}} p_{2}^{a_{2}} \partial_{w_{1}}\left\{\frac{\eta^{a_{3} b_{1}} \eta^{b_{2} m_{1}}}{\left(z_{1}-z_{2}\right)^{2}\left(z_{2}-\bar{z}\right)^{2}}\left(\frac{1}{z_{1}-z_{2}}-\frac{1}{z_{1}-\bar{z}}-\frac{1}{z_{1}-z}\right)^{2}\right\}\right. \\
-p_{2}^{a_{1}} p_{1}^{b_{1}} \partial_{w_{1}}\left\{\frac{\eta^{a_{2} b_{2}} \eta^{a_{3} m_{1}}}{\left(z_{1}-z_{2}\right)^{2}\left(z_{1}-\bar{z}\right)^{2}}\left(\frac{1}{z_{1}-z_{2}}-\frac{1}{z_{1}-\bar{z}}-\frac{1}{z_{1}-z}\right)\right. \\
\left.\times\left(\frac{1}{z_{1}-z_{2}}+\frac{1}{z_{2}-\bar{z}}+\frac{1}{z_{2}-z}\right)\right\} \\
-p_{2}^{a_{1}} p_{1}^{m_{1}} \partial_{w_{1}}\left\{\frac{\eta^{a_{2} b_{1}} \eta^{a_{3} b_{2}}}{\left(z_{1}-z_{2}\right)^{4}}\left(\frac{1}{z_{1}-z_{2}}-\frac{1}{z_{1}-\bar{z}}-\frac{1}{z_{1}-z}\right)\right. \\
\left.\left.\times\left(\frac{1}{z_{1}-z_{2}}+\frac{1}{z_{2}-\bar{z}}+\frac{1}{z_{2}-z}\right)\left(-\frac{1}{z_{1}-\bar{z}}+\frac{1}{z_{2}-\bar{z}}\right)\right\}\right\}
\end{array}
$$


Next,

$$
\begin{array}{r}
<A_{4}\left(z_{1}\right) A_{0}\left(z_{2}\right) V_{s=2}(z, \bar{z})>_{2}=-2 H_{a_{1} a_{2} a_{3}}\left(p_{1}\right) H_{b_{1} b_{2} b_{3}} \gamma_{m_{1} m_{2}} \\
\times \int_{-\infty}^{\infty} d z_{1} \int_{-\infty}^{\infty} d z_{2}\left(z_{1}-i\right)^{2}\left(z_{2}+i\right)^{2} G_{g h}\left(z_{1}, z_{2}, i,-i\right)\left(-\frac{1}{z_{1}-z_{2}}+\frac{2}{z_{1}-\bar{z}}+\frac{1}{z_{1}-z}\right) \\
\times\left(-\frac{\eta^{a_{3} b_{3}} \eta^{a_{2} m_{2}}}{\left(z_{1}-z_{2}\right)\left(z_{1}-z\right)^{2}}+\frac{\eta^{a_{2} b_{2}} \eta^{a_{3} m_{2}}}{\left(z_{1}-z_{2}\right)^{2}\left(z_{1}-z\right)}\right) \\
\times\left\{-p_{1}^{b_{1}} p_{2}^{a_{1}}\left(\frac{1}{z_{1}-z_{2}}-\frac{1}{z_{1}-\bar{z}}-\frac{1}{z_{1}-z}\right)\left(\frac{1}{z_{1}-z_{2}}+\frac{1}{z_{2}-\bar{z}}+\frac{1}{z_{2}-z}\right) \frac{\eta^{b_{2} m_{1}}}{\left(z_{2}-\bar{z}\right)^{2}}\right. \\
+-p_{1}^{b_{1}} p_{1}^{b_{2}}\left(\frac{1}{z_{1}-z_{2}}+\frac{1}{z_{2}-\bar{z}}+\frac{1}{z_{2}-z}\right)^{2} \frac{\eta^{a_{1} m_{1}}}{\left(z_{1}-\bar{z}\right)^{2}} \\
\left.-p_{1}^{b_{1}} p_{1}^{m_{1}}\left(\frac{1}{z_{2}-\bar{z}}-\frac{1}{z_{1}-\bar{z}}\right)\left(\frac{1}{z_{1}-z_{2}}+\frac{1}{z_{2}-\bar{z}}+\frac{1}{z_{2}-z}\right) \frac{\eta^{a_{1} b_{2}}}{\left(z_{1}-z_{2}\right)^{2}}\right\}
\end{array}
$$

Next,

$$
\begin{array}{r}
<A_{5}(i) A_{0}(-i) V_{s=2}(z, \bar{z})>_{2}=-2 H_{a_{1} a_{2} a_{3}}\left(p_{1}\right) H_{b_{1} b_{2} b_{3}}\left(p_{2}\right) \gamma_{m_{1} m_{2}}\left(p_{3}\right) \\
\times \int_{-\infty}^{\infty} d z_{1} \int_{-\infty}^{\infty} d z_{2}\left(z_{1}-i\right)^{2}\left(z_{2}+i\right)^{2} G_{g h}\left(z_{1}, z_{2}, i,-i\right) \\
\times\left(\frac{\eta^{a_{3} b_{3}} \eta^{a_{2} m_{2}}}{\left(z_{1}-z_{2}\right)\left(z_{1}-z\right)^{2}}\left(\frac{1}{z_{1}-z_{2}}+\frac{2}{w_{2}-z}\right)-\frac{\eta^{a_{2} b_{2}} \eta^{a_{3} m_{2}}}{\left(z_{1}-z_{2}\right)^{2}\left(z_{1}-z\right)}\right)\left(\frac{2}{z_{1}-z_{2}}+\frac{1}{w_{2}-z}\right) \\
\times\left\{-p_{1}^{b_{1}} p_{2}^{a_{1}}\left(\frac{1}{z_{1}-z_{2}}-\frac{1}{z_{1}-\bar{z}}-\frac{1}{z_{1}-z}\right)\left(\frac{1}{z_{1}-z_{2}}+\frac{1}{z_{2}-\bar{z}}+\frac{1}{z_{2}-z}\right) \frac{\eta^{b_{2} m_{1}}}{\left(z_{2}-\bar{z}\right)^{2}}\right. \\
+-p_{1}^{b_{1}} p_{1}^{b_{2}}\left(\frac{1}{z_{1}-z_{2}}+\frac{1}{z_{2}-\bar{z}}+\frac{1}{z_{2}-z}\right)^{2} \frac{\eta^{a_{1} m_{1}}}{\left(z_{1}-\bar{z}\right)^{2}} \\
\left.-p_{1}^{b_{1}} p_{1}^{m_{1}}\left(\frac{1}{z_{2}-\bar{z}}-\frac{1}{z_{1}-\bar{z}}\right)\left(\frac{1}{z_{1}-z_{2}}+\frac{1}{z_{2}-\bar{z}}+\frac{1}{z_{2}-z}\right) \frac{\eta^{a_{1} b_{2}}}{\left(z_{1}-z_{2}\right)^{2}}\right\}
\end{array}
$$

Next,

$$
\begin{array}{r}
<A_{6}(i) A_{0}(-i) V_{s=2}(z, \bar{z})>_{2}=H_{a_{1} a_{2} a_{3}}\left(p_{1}\right) H_{b_{1} b_{2} b_{3}}\left(p_{2}\right) \gamma_{m_{1} m_{2}}\left(p_{3}\right) \\
\times \int_{-\infty}^{\infty} d z_{1} \int_{-\infty}^{\infty} d z_{2}\left(z_{1}-i\right)^{2}\left(z_{2}+i\right)^{2} G_{g h}\left(z_{1}, z_{2}, i,-i\right)\left(-\frac{1}{z_{1}-z_{2}}+\frac{2}{z_{1}-\bar{z}}+\frac{1}{z_{1}-z}\right) \\
\times \frac{\left(p_{1}\right)_{\alpha_{4}}}{\left(z_{1}-z_{2}\right)\left(z_{1}-z\right)}\left(-\eta^{a_{3} b_{3}} \eta^{a_{4} m_{2}}+\eta^{a_{4} b_{3}} \eta^{a_{3} m_{2}}\right) \\
\times\left\{\frac{p_{2}^{a_{1}} \eta^{a_{2} b_{1}} \eta^{b_{2} m_{1}}}{\left(z_{1}-z_{2}\right)^{2}\left(z_{2}-\bar{z}\right)^{2}}\left(\frac{1}{z_{1}-z_{2}}-\frac{1}{z_{1}-\bar{z}}-\frac{1}{z_{1}-z}\right)\right. \\
-\frac{p_{1}^{b_{1}} \eta^{a_{1} b_{2}} \eta^{a_{2} m_{1}}}{\left(z_{1}-z_{2}\right)^{2}\left(z_{1}-\bar{z}\right)^{2}}\left(\frac{1}{z_{1}-z_{2}}+\frac{1}{z_{2}-\bar{z}}+\frac{1}{z_{2}-z}\right) \\
\left.+\frac{p_{1}^{m_{1}} \eta^{a_{1} b_{1}} \eta^{a_{2} b_{2}}}{\left(z_{1}-z_{2}\right)^{4}}\left(\frac{1}{z_{2}-\bar{z}}+\frac{1}{z_{1}-\bar{z}}\right)\right\}
\end{array}
$$


Finally,

$$
\begin{array}{r}
<A_{7}(i) A_{0}(-i) V_{s=2}(z, \bar{z})>_{2}=H_{a_{1} a_{2} a_{3}}\left(p_{1}\right) H_{b_{1} b_{2} b_{3}}\left(p_{2}\right) \gamma_{m_{1} m_{2}}\left(p_{3}\right) \\
\times \int_{-\infty}^{\infty} d z_{1} \int_{-\infty}^{\infty} d z_{2}\left(z_{1}-i\right)^{2}\left(z_{2}+i\right)^{2} G_{g h}\left(z_{1}, z_{2}, i,-i\right) \\
\quad \times \frac{\left(p_{1}\right)_{\alpha_{4}}}{\left(z_{1}-z_{2}\right)\left(z_{1}-z\right)}\left(-\frac{\eta^{a_{3} b_{3}} \eta^{a_{4} m_{2}}}{z_{1}-z}+\frac{\eta^{a_{4} b_{3}} \eta^{a_{3} m_{2}}}{z_{1}-z_{2}}\right) \\
\times\left\{\frac{p_{2}^{a_{1}} \eta^{a_{2} b_{1}} \eta^{b_{2} m_{1}}}{\left(z_{1}-z_{2}\right)^{2}\left(z_{2}-\bar{z}\right)^{2}}\left(\frac{1}{z_{1}-z_{2}}-\frac{1}{z_{1}-\bar{z}}-\frac{1}{z_{1}-z}\right)\right. \\
-\frac{p_{1}^{b_{1}} \eta^{a_{1} b_{2}} \eta^{a_{2} m_{1}}}{\left(z_{1}-z_{2}\right)^{2}\left(z_{1}-\bar{z}\right)^{2}}\left(\frac{1}{z_{1}-z_{2}}+\frac{1}{z_{2}-\bar{z}}+\frac{1}{z_{2}-z}\right) \\
\left.+\frac{p_{1}^{m_{1}} \eta^{a_{1} b_{1}} \eta^{a_{2} b_{2}}}{\left(z_{1}-z_{2}\right)^{4}}\left(\frac{1}{z_{2}-\bar{z}}+\frac{1}{z_{1}-\bar{z}}\right)\right\}
\end{array}
$$

This concludes the list of terms,quadratic in momentum. Finally, we shall list the contributions of the order zero in momentum. We obtain

$$
\begin{array}{r}
<A_{1}(i) A_{0}(-i) V_{s=2}(z, \bar{z})>_{0}=-H_{a_{1} a_{2} a_{3}}\left(p_{1}\right) H_{b_{1} b_{2} b_{3}}\left(p_{2}\right) \gamma_{m_{1} m_{2}}\left(p_{3}\right) \\
\times \int_{-\infty}^{\infty} d z_{1} \int_{-\infty}^{\infty} d z_{2}\left(z_{1}-i\right)^{2}\left(z_{2}+i\right)^{2} G_{g h}\left(z_{1}, z_{2}, i,-i\right) \\
\quad \times\left\{\frac{\left(\eta^{a_{4} b_{3}} \eta^{a_{3} m_{2}}-\eta^{a_{3} b_{3}} \eta^{a_{4} m_{2}}\right) \eta_{a_{4}}^{b_{2}} \eta^{a_{1} b_{1}} \eta^{a_{2} m_{1}}}{\left(z_{1}-z_{2}\right)^{5}\left(z_{1}-\bar{z}\right)^{2}\left(z_{1}-z\right)}\right\}
\end{array}
$$

Then,

$$
\begin{aligned}
& <A_{2}(i) A_{0}(-i) V_{s=2}(z, \bar{z})>_{0}=-H_{a_{1} a_{2} a_{3}}\left(p_{1}\right) H_{b_{1} b_{2} b_{3}}\left(p_{2}\right) \gamma_{m_{1} m_{2}}\left(p_{3}\right) \\
& \times \int_{-\infty}^{\infty} d z_{1} \int_{-\infty}^{\infty} d z_{2}\left(z_{1}-i\right)^{2}\left(z_{2}+i\right)^{2} G_{g h}\left(z_{1}, z_{2}, i,-i\right)\left(-\frac{1}{z_{1}-z_{2}}+\frac{2}{z_{1}-\bar{z}}+\frac{1}{z_{1}-z}\right) \\
& \times \frac{\eta^{a_{1} b_{1}} \eta^{a_{2} b_{2}} \eta^{a_{3} m_{1}} \eta^{b_{3} m_{2}}}{\left(z_{1}-z_{2}\right)^{4}\left(z_{1}-\bar{z}\right)^{2}\left(z_{1}-z\right)}
\end{aligned}
$$

Next,

$$
\begin{aligned}
& <A_{3}(i) A_{0}(-i) V_{s=2}(z, \bar{z})>_{0}=-H_{a_{1} a_{2} a_{3}}\left(p_{1}\right) H_{b_{1} b_{2} b_{3}}\left(p_{2}\right) \gamma_{m_{1} m_{2}}\left(p_{3}\right) \\
& \times \int_{-\infty}^{\infty} d z_{1} \int_{-\infty}^{\infty} d z_{2}\left(z_{1}-i\right)^{2}\left(z_{2}+i\right)^{2} G_{g h}\left(z_{1}, z_{2}, i,-i\right) \\
& \times \partial_{w_{1}}\left\{\frac{\eta^{a_{1} b_{1}} \eta^{a_{2} b_{2}} \eta^{a_{3} m_{1}} \eta^{b_{3} m_{2}}}{\left(z_{1}-z_{2}\right)^{4}\left(z_{1}-\bar{z}\right)^{2}\left(z_{1}-z\right)}\right\}
\end{aligned}
$$


Next,

$$
\begin{array}{r}
<A_{4}(i) A_{0}(-i) V_{s=2}(z, \bar{z})>_{0}=-4 H_{a_{1} a_{2} a_{3}}\left(p_{1}\right) H_{b_{1} b_{2} b_{3}}\left(p_{2}\right) \gamma_{m_{1} m_{2}}\left(p_{3}\right) \\
\times \int_{-\infty}^{\infty} d z_{1} \int_{-\infty}^{\infty} d z_{2}\left(z_{1}-i\right)^{2}\left(z_{2}+i\right)^{2} G_{g h}\left(z_{1}, z_{2}, i,-i\right)\left(-\frac{1}{z_{1}-z_{2}}+\frac{2}{z_{1}-\bar{z}}+\frac{1}{z_{1}-z}\right) \\
\times \frac{\eta^{a_{1} b_{1}} \eta^{b_{2} m_{2}}}{\left(z_{1}-z_{2}\right)^{2}\left(z_{2}-z\right)^{2}}\left(\frac{\eta^{a_{2} m_{1}} \eta^{a_{3} b_{3}}}{\left(z_{1}-\bar{z}\right)^{2}\left(z_{1}-z_{2}\right)}-\frac{\eta^{a_{2} b_{3}} \eta^{a_{3} m_{1}}}{\left(z_{1}-\bar{z}\right)\left(z_{1}-z_{2}\right)^{2}}\right)
\end{array}
$$

Finally,

$$
\begin{array}{r}
<A_{5}(i) A_{0}(-i) V_{s=2}(z, \bar{z})>_{0}=-2 H_{a_{1} a_{2} a_{3}}\left(p_{1}\right) H_{b_{1} b_{2} b_{3}}\left(p_{2}\right) \gamma_{m_{1} m_{2}}\left(p_{3}\right) \\
\times \int_{-\infty}^{\infty} d z_{1} \int_{-\infty}^{\infty} d z_{2}\left(z_{1}-i\right)^{2}\left(z_{2}+i\right)^{2} G_{g h}\left(z_{1}, z_{2}, i,-i\right) \\
\times \partial_{w_{1}}\left\{\frac{\eta^{a_{1} b_{1}} \eta^{b_{2} m_{2}}}{\left(z_{1}-z_{2}\right)^{2}\left(z_{2}-z\right)^{2}}\left(\frac{\eta^{a_{2} m_{1}} \eta^{a_{3} b_{3}}}{\left(z_{1}-\bar{z}\right)^{2}\left(z_{1}-z_{2}\right)}-\frac{\eta^{a_{2} b_{3}} \eta^{a_{3} m_{1}}}{\left(z_{1}-\bar{z}\right)\left(z_{1}-z_{2}\right)^{2}}\right)\right\} \\
<A_{6}(i) A_{0}(-i) V_{s=2}(z, \bar{z})>_{0}=<A_{7}(i) A_{0}(-i) V_{s=2}(z, \bar{z})>_{0}=0
\end{array}
$$

This concludes the list of all the contributions to the spin 3-graviton scattering, up to the terms, quartic in momentum. The next step is to evaluate the $z_{1}, z_{2}$-integrals , giving the relative coefficients in front of all the terms, listed in (27)-(45). To perform the integration, it is convenient to introduce the regulator $e^{i \lambda\left(z_{1}+z_{2}\right)}(\lambda>0)$ inside each of the integrals (setting $\lambda \rightarrow 0$ upon the calculation). The regulator ensures that the contour integrals over cemicircle of radius $R \rightarrow \infty$ in the upper half-plane vanish and therefore the straight line integrals can be obtained from the the appropriate residues in the $z_{1}, z_{2}$ integrals. Shifting the straight line $z_{1}, z_{2}$ contours according to $z_{1} \rightarrow z_{1}-i 0, z_{2} \rightarrow z_{2}+i 0$, it is convenient to evaluate the integral in $z_{2}$ first and then in $z_{1}$. The evaluation of the $z_{1}, z_{2}$ integrals is then straightforward; transforming back to the position space we find that the total expression for the three-vertex of two $s=3$ particles and a graviton from 
the three-point amplitude on the disc is given by:

$$
\begin{aligned}
& -4 \pi^{2} A_{s=3,3,2}=-2 \eta^{a_{3} b_{3}} \eta^{a_{1} m_{1}} \partial_{b_{1}} \partial_{b_{2}} \partial_{m_{2}} H^{a_{1} a_{2} a_{3}} \partial_{a_{2}} H^{b_{1} b_{2} b_{3}} \gamma^{m_{1} m_{2}} \\
& +2 \eta_{a_{1} m_{1}} \eta_{a_{2} m_{2}} \eta^{a_{4} b_{4}} \partial_{b_{1}} \partial_{b_{2}} \partial_{b_{4}} H^{a_{1} a_{2} a_{3}} \partial_{a_{4}} H^{b_{1} b_{2} b_{3}} \gamma^{m_{1} m_{2}} \\
& +2 \eta^{a_{1} m_{1}} \eta^{a_{2} m_{2}} \partial_{b_{1}} \partial_{b_{2}} \partial_{b_{3}} H^{a_{1} a_{2} a_{3}} \partial_{a_{3}} H^{b_{1} b_{2} b_{3}} \gamma^{m_{1} m_{2}} \\
& +\eta_{a_{2} b_{2}} \eta_{a_{3} b_{3}} \partial_{b_{1}} \partial_{m_{1}} \partial_{m_{2}} H^{a_{1} a_{2} a_{3}} \partial_{a_{1}} H^{b_{1} b_{2} b_{3}} \gamma^{m_{1} m_{2}} \\
& -2 \eta_{a_{1} m_{1}} \eta_{a_{2} b_{3}} \partial_{b_{1}} \partial_{b_{2}} \partial_{m_{2}} H^{a_{1} a_{2} a_{3}} \partial_{a_{3}} H^{b_{1} b_{2} b_{3}} \gamma^{m_{1} m_{2}} \\
& -2 \eta_{a_{2} m_{1}} \eta_{b_{2} m_{2}} \partial_{b_{1}} \partial_{b_{3}} H^{a_{1} a_{2} a_{3}} \partial_{a_{1}} \partial_{a_{3}} H^{b_{1} b_{2} b_{3}} \gamma^{m_{1} m_{2}} \\
& -2 \eta_{a_{2} m_{1}} \eta_{b_{2} m_{2}} \eta_{a_{1} b_{3}} \eta^{a_{4} b_{4}} \partial_{b_{1}} \partial_{a_{4}} H^{a_{1} a_{2} a_{3}} \partial_{a_{3}} \partial_{b_{4}} H^{b_{1} b_{2} b_{3}} \gamma^{m_{1} m_{2}} \\
& +\eta_{a_{2} b_{2}} \eta_{a_{3} b_{3}} \eta_{b_{1} m_{1}} \eta^{a_{4} b_{4}} \partial_{m_{2}} \partial_{a_{4}} H^{a_{1} a_{2} a_{3}} \partial_{a_{1}} \partial_{b_{4}} H^{b_{1} b_{2} b_{3}} \gamma^{m_{1} m_{2}} \\
& +2 \eta_{a_{1} m_{1}} \eta_{a_{2} m_{2}} \eta_{a_{3} b_{2}} \eta^{a_{4} b_{4}} \partial_{b_{1}} \partial_{b_{3}} \partial_{a_{4}} H^{a_{1} a_{2} a_{3}} \partial_{b_{4}} H^{b_{1} b_{2} b_{3}} \gamma^{m_{1} m_{2}} \\
& -2 \eta_{a_{1} m_{1}} \eta_{a_{2} m_{2}} \eta_{a_{3} b_{1}} \eta^{a_{4} b_{4}} \partial_{b_{2}} \partial_{a_{4}} H^{a_{1} a_{2} a_{3}} \partial_{a_{2}} \partial_{b_{4}} H^{b_{1} b_{2} b_{3}} \gamma^{m_{1} m_{2}} \\
& +\eta_{a_{1} m_{2}} \eta_{a_{2} b_{2}} \eta_{a_{3} b_{3}} \eta^{a_{4} b_{4}} \partial_{b_{1}} \partial_{m_{1}} \partial_{a_{4}} H^{a_{1} a_{2} a_{3}} \partial_{b_{4}} H^{b_{1} b_{2} b_{3}} \gamma^{m_{1} m_{2}} \\
& -\eta_{a_{1} m_{1}} \eta_{b_{3} m_{2}} \partial_{b_{1}} \partial_{b_{2}} H^{a_{1} a_{2} a_{3}} \partial_{a_{2}} \partial_{a_{3}} H^{b_{1} b_{2} b_{3}} \gamma^{m_{1} m_{2}} \\
& -2 \eta_{b_{1} m_{1}} \eta_{a_{2} m_{2}} \eta_{a_{3} b_{2}} \eta^{a_{4} b_{4}} \partial_{b_{3}} \partial_{a_{4}} H^{a_{1} a_{2} a_{3}} \partial_{a_{1}} \partial_{b_{4}} H^{b_{1} b_{2} b_{3}} \gamma^{m_{1} m_{2}} \\
& +2 \eta_{a_{1} m_{1}} \eta_{a_{2} m_{2}} \eta_{a_{3} b_{3}} \eta^{a_{4} b_{4}} \partial_{b_{1}} \partial_{b_{2}} \partial_{a_{4}} H^{a_{1} a_{2} a_{3}} \partial_{b_{4}} H^{b_{1} b_{2} b_{3}} \gamma^{m_{1} m_{2}} \\
& +2 \eta_{a_{2} m_{1}} \eta_{a_{3} m_{2}} \partial_{b_{1}} \partial_{b_{2}} \partial_{b_{3}} H^{a_{1} a_{2} a_{3}} \partial_{a_{1}} H^{b_{1} b_{2} b_{3}} \gamma^{m_{1} m_{2}} \\
& +2 \eta_{a_{1} m_{1}} \eta_{b_{1} m_{2}} \eta_{a_{2} b_{2}} \eta_{a_{3} b_{3}} \eta^{a_{4} b_{4}} \eta^{a_{5} b_{5}} \partial_{a_{4}} \partial_{a_{5}} H^{a_{1} a_{2} a_{3}} \partial_{b_{4}} \partial_{b_{5}} H^{b_{1} b_{2} b_{3}} \gamma^{m_{1} m_{2}}
\end{aligned}
$$

Contracting the indices and using the conditions (2), it is straightforward to show that, modulo partial integration one can cast the expression (46) as

$$
=w_{a b c d}\left\{2 h_{m n}^{a} \partial^{d} \partial^{n} h^{b c m}-2 \partial^{m} h^{a c n} \partial_{m} h_{n}^{b d}-2 \partial^{n} h^{a c m} \partial_{m} h^{b d}-h_{m n}^{a} \partial^{b} \partial^{d} h^{c m n}\right\}
$$

where $w_{a b c d}(\gamma)$ is linearized Weyl tensor. Remarkably, the terms (34)-(40), quadratic in derivatives, as well as those of (41)-(45) containing no derivatives, drop out as a result of the integration, so only the terms, quartic in derivatives remain. It is instructive to compare the expressions $(46),(47)$ to the interaction vertex given in the important papers [21], [22] (this vertex is also a flat limit of the one found in [1] for the AdS case) According to [21], [22], the interaction of graviton with massless spin 3 fields is determined by the linearized Weyl tensor times the quadratic combination of spin 3 fields. The 3 -vertex (46), (47) following from the string scattering amplitude on the disc, reproduces the 3-vertex 
found in [21], 222] with the gauge partially fixed according to the constraints (2). The fact that string theory gives us the gauge fixed version of the cubic vertex of the spin 3 graviton interaction, is not surprising, if we compare the higher spin case to the standard example of a photon when the BRST (transversality) constraints on the vertex operator naturally imply the Lorenz gauge choice for the space-time field.

\section{Conclusion and Discussion}

In this paper we have determined the gravitational coupling of the massless spin 3 field from string theory, by computing the appropriate correlation function on the disc. Remarkably, the string theory calculation turns out to reproduce (up to partial gauge fixing) the non-Abelian cubic coupling of the spin 3 field with the graviton through the linearized Weyl tensor, derived in [21], [22], which also is the flat limit of the FradkinVasiliev vertex in the frame-like approach [1] This result is in agreement with more general property of spin $s$ fields coupling to the gravity, with the non-Abelian cubic interaction vertices containing not less than $2 s-2$ space-time derivatives. Note that, although the lower derivative terms initially appear in the calculation, they all drop out as a result of the integration (recall that the positive picture expressions for the higher spin operators exist in the integrated form only) This altogether is an important consistency test for the string vertex operators for the higher spins, considered in this work. It would be important to generalize this disc calculation to particles with the spins $s \geq 4$ interacting with the graviton, in order to check the $2 s-2$ derivative rule for $s>3$. Generally, one would expect each of the correlators to produce three principal contributions, differing in the number of the space-time derivatives $(2 s-2,2 s$ and $2 s+2)$, according to corresponding to three possible cubic couplings of spin $s$ particles and the graviton [7]. Although we concentrated on the 3-point amplitude of two spin 3 particles with the graviton on the disc, the amplitude, considered in this paper is structurally similar to the 4-point amplitude in open string theory, involving two photons and 2 spin 3 particles. Generally, the quartic terms in the interacting higher spin field theories are cumbersome and their structure is not yet well understood. It would be important to investigate the quartic couplings by using the vertex operator computations in string theory, which appears to be an efficient and a promising framework to approach the problem.

\section{Acknowledgements}

I would like express my gratitude to Augusto Sagnotti and other members of the group at SNS in Pisa for the hospitality and the productive discussions during my visit to Scuola Normale in January 2010, where the initial stages of this work were completed. 
It is a pleasure to thank Nick Boulanger and Per Sundell for illuminating and productive discussions on the vertex operators and the non-Abelian couplings for the higher spin fields. In particular, I would like to thank Per Sundell for pointing out to me a number of interesting problems related to non-Abelian couplings of the higher spin fields. I also would like to thank Yoshihisa Kitazawa for the hospitality during my visit to KEK in February 2010, where parts of the calculation, presented in this work, have been done. 


\section{References}

[1] E. Fradkin, M. Vasiliev, Phys. Lett. B189 (1987) 89

[2] M. Vasiliev, Phys. Lett. B243 (1990) 378

[3] M. Vasiliev, Int. J. Mod. Phys. D5 (1996) 763

[4] A. Bengtsson, I. Bengtsson, L. Brink, Nucl. Phys. B227 (1983) 31

[5] S. Deser, Z. Yang, Class. Quant. Grav 7 (1990) 1491

[6] X. Bekaert, N. Boulanger, S. Cnockaert, J. Math. Phys 46 (2005) 012303

[7] R. Metsaev, arXiv:0712.3526

[8] W. Siegel, B. Zwiebach, Nucl. Phys. B282 (1987) 125

[9] A. Neveu, H. Nicolai, P. West, Nucl. Phys. B264 (1986) 573

[10] T. Damour, S. Deser, Ann. Poincare Phys. Theor. 47 (1987) 277

[11] D. Francia, A. Sagnotti, Phys. Lett. B53 (2002) 303

[12] A. Sagnotti, E. Sezgin, P. Sundell, hep-th/0501156

[13] L. Brink, R.Metsaev, M. Vasiliev, Nucl. Phys. B 586 (2000)183

[14] X. Bekaert, S. Cnockaert, C. Iazeolla, M.A. Vasiliev, IHES-P-04-47, ULB-TH-04-26, ROM2F-04-29, FIAN-TD-17-04, Sep 2005 86pp.

[15] A. Campoleoni, D. Francia, J. Mourad, A. Sagnotti, Nucl. Phys. B815 (2009) 289-367

[16] A. Campoleoni, D. Francia, J. Mourad, A. Sagnotti, arXiv:0904.4447

[17] D. Francia, A. Sagnotti, J.Phys.Conf.Ser.33:57 (2006)

[18] M. Taronna, arXiv:1005.3061

[19] D. Polyakov, arXiv:0910.5338

[20] C. Fronsdal, Phys. Rev. D18 (1978) 3624

[21] N. Boulanger, S. Leclercq, JHEP 11 (2006) 034

[22] N. Boulanger, S. Leclercq, P. Sundell, JHEP 0808 (2008) 056

[23] G. Barnich, M. Henneaux, Phys. Lett. B311 (1993) 123-129

[24] M. Henneau, Contemp. Math. 219 (1998) 93

[25] E.S. Fradkin, M. A. Vasiliev, Nucl. Phys. B291 (1987) 141

[26] C. Aragone, S. Deser, Nuovo Cim. B57 (1980) 33-49

[27] F. Berends, J. W. van Holten, P. van Nieuwenhuizen, B. de Wit, Phys. Lett. B83 (1979) 188

[28] R. Metsaev, Nucl. Phys. B759 (2006) 147-201

[29] D. Polyakov, arXiv:0905.4858

[30] D. Polyakov, arXiv:0906.3663

[31] D. Polyakov, Int.J.Mod.Phys.A20:4001-4020,2005 